inflammatory neurological diseases. The levels of albumin and IL-6 in CSF and sera were measured by ELISA.

Results: Serum IL- 6 as well as CSF IL- 6 was significantly elevated in acute confusional state (ACS) compared with non-ACS diffuse NPSLE (anxiety disorder, cognitive dysfunction, mood disorder and psychosis) or focal NPSLE (figure). Q albumin (CSF/serum albumin quotient) was also significantly higher in ACS than in the other 2 groups of NPSLE. Of note, serum IL-6 $(r=0.2801, p=0.0207)$, but not CSF IL-6 $(r=0.1602, p=0.1918)$, was significantly correlated with $Q$ albumin in patients with diffuse NPSLE, including ACS and non-ACS, although serum IL-6 was significantly correlated with CSF IL-6 in this population $(r=0.3205, p=0.082)$.

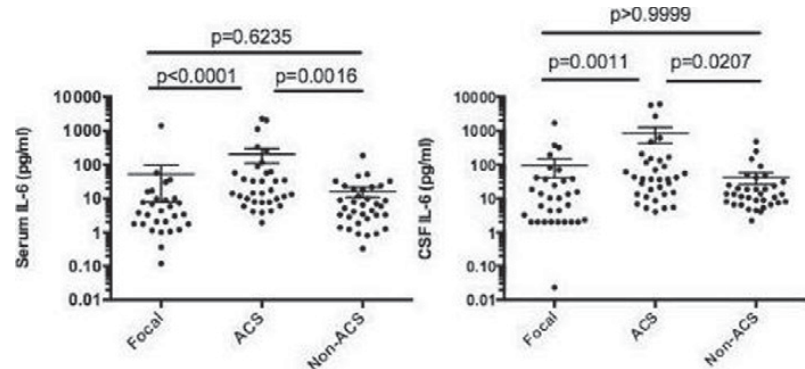

Conclusions: These results indicate that serum IL- 6 as well as IL-6 is involved in the pathogenesis of NPSLE. The data also confirm that the severity of BBB damages plays a crucial role in the development of ACS, the severest form of diffuse NPSLE. Finally, it is suggested that serum IL-6 might play a most important role in BBB breakdown in NPSLE, whereas CSF IL-6 might be involved in subsequent central nervous system inflammation.

Disclosure of Interest: None declared

DOI: 10.1136/annrheumdis-2017-eular.2806

\section{FRI0298 APPLICATION OF PROPENSITY SCORE-MATCHING METHODS TO COMPARE DATA FROM LONG-TERM EXTENSION TRIALS WITH DATA FROM AN EXISTING LUPUS REGISTRY}

M.B. Urowitz ${ }^{1}$, R. Wielage ${ }^{2}$, K.A. Kelton ${ }^{2}$, R.L. Ohsfeldt ${ }^{2}$, Y. Asukai ${ }^{3}$, S. Ramachandran ${ }^{4} .{ }^{1}$ Centre for Prognosis Studies in Rheumatic Diseases, Toronto Western Hospital, Toronto, Canada; ${ }^{2}$ Medical Decision Modeling Inc., Indianapolis, United States; ${ }^{3}$ Value Evidence \& Outcomes, GSK, Stockley Park, United Kingdom; ${ }^{4}$ Value Evidence \& Outcomes, GSK, Philadelphia, United States

Background: Phase III randomised controlled trials (BLISS 52 and 76) in patients with systemic lupus erythematosus (SLE) have established the efficacy and safety of belimumab plus standard SLE therapy (SoC) vs SoC alone. As with many continuation studies, the long-term extensions (LTE) of the BLISS trials (BEL112233/NCT00724867 and BEL112234/NCT00712933) were single-arm, open-label studies; therefore, comparative long-term efficacy of belimumab and SoC vs SoC was not assessed. Existing observational databases could provide a valuable source of comparison data.

Objectives: This study (BEL206347) explores the use of propensity score (PS) adjustment to enable a post-hoc comparison of LTE patients to patients from an existing SLE cohort, assessing long-term efficacy of belimumab plus SoC vs SoC using the SLICC/ACR Damage Index (SDI) as the outcome.

Methods: Potential PS model criteria were identified by a systematic literature review for factors predicting organ damage. PS model criteria were restricted to baseline (at index [first recorded disease activity of SLE Disease Activity Index $\{$ SLEDAI $\geq 6$ ] for SoC cohort) characteristics available for both cohorts, including age, gender, race, disease duration, clinical and laboratory characteristics, SLEDAl over time, baseline SLEDAI and SDI and SoC (e.g. corticosteroid use/dose). Patients with active severe lupus nephritis/central nervous system lupus, those who had received B-cell-targeted therapy or belimumab or those with an index date before 1990 were excluded from the SoC cohort. The primary PS procedure used 1:1 matching with a $20 \%$ calliper (PSM); alternatively, inverse PS weighting (IPSW) was used to preserve the LTE sample. The balance in baseline factors across cohorts pre-/post-PS-adjustment was assessed using standardised distance measures.

Results: The literature review identified the Toronto Lupus Cohort (TLC) as an external registry with a comparable patient cohort. Without PS-adjustment, the standardised distance across cohorts exceeded $25 \%$ for 13 of 17 baseline factors in the PSM model. After PSM, standardised distance was $<10 \%$ for all factors, but less than half of the LTE patients were matched. Using IPSW, data from all patients could be used but with a weighted standardised distance of $<10 \%$ for 8 and $<25 \%$ for 15 of the 17 PSM model factors.

Conclusions: The TLC was identified as an existing lupus registry with the endpoints of interest and detailed clinical characteristics. PS matching further maximised the comparability of the LTE and TLC cohorts. The final predictors used in the matching will be validated by two SLE clinicians. The preliminary results indicate a statistically adequate balance in clinical characteristics is attainable. PS matching will allow comparison of long-term organ damage in patients with SLE receiving belimumab plus SoC vs SoC alone. This approach can be applied to other extension studies to assess long-term treatment effectiveness.
Acknowledgements: Study funded by GSK. Katie White, PhD, Fishawack Indicia Ltd, provided editorial assistance funded by GSK.

Disclosure of Interest: M. Urowitz Grant/research support from: GSK, UCB and Eli Lilly, Consultant for: GSK, UCB and Eli Lilly, R. Wielage Consultant for: GSK, K. Kelton Consultant for: GSK, R. Ohsfeldt Consultant for: GSK, Amgen and AstraZeneca Hygieia, Y. Asukai Shareholder of: GSK, Employee of: GSK, S. Ramachandran Shareholder of: GSK, Employee of: GSK

DOI: 10.1136/annrheumdis-2017-eular.5071

\section{FRI0299 N-TERMINAL PRO-BRAIN NATRIURETIC PEPTIDE (NT-PROBNP) LEVEL IN PATIENTS WITH UNTREATED SYSTEMIC LUPUS ERYTHEMATOSUS}

T. Panafidina, M. Sokhova, T. Popkova, D. Novikova, E. Alexandrova, E. Nasonov. V.A.Nasonova Research Institute of Rheumatology, Moscow, Russian Federation

Objectives: The aim of this study was to determine NT-proBNP serum levels in patients with untreated SLE, thus ruling out any potential effect of SLE therapy: to analyze any possible correlation between NT-proBNP values and traditional risk factors (TRF), inflammatory markers and myocardial function parameters.

Methods: The study included 28 pts (82\% females, aged $28,5[25,0-32,0]$ years (median [interquartile range $25 \%>75 \%$ ]) with untreated SLE (ACR criteria, $1997)$ and 27 healthy controls (89\% females, age 30,0 [23,0-49,0] years). None of SLE pts was treated either with prednisone or cytotoxic drugs at the moment of inclusion, 5 (18\%) pts received hydroxychloroquine $200 \mathrm{mg} /$ day. SLE-related factors, including disease duration, clinical features, SLE Disease Activity Index (SLEDAI 2K) and Systemic Lupus International Collaborating Clinics damage index (SLICC/DI) were evaluated in parallel with relevant laboratory findings (blood and urine tests, CRP, IL-6, INF- $\alpha$, immunoglobulins $\mathrm{G}, \mathrm{A}$ and $\mathrm{M}, \mathrm{C} 3$ and $\mathrm{C} 4$ complement fragments and others), autoantibodies (ANA, antiDNA, ENA-SSA, -SSB, -Sm, -RNP-70, aPL), echocardiography was performed using standard techniques. Serum levels of NT-proBNP $(\mathrm{pg} / \mathrm{ml})$ were measured using electrochemiluminescence method Elecsys proBNP II (Roche Diagnostics, Switzerland). Normal NT-proBNP levels should vary within $\leq 125,0$ $\mathrm{pg} / \mathrm{mL}$.

Results: Mean SLE duration was 21,0 [5,0-60,0] months, SLEDAI 2K score - 11 [8-9], SLICC/DI score - $0[0-0]$. SLE pts had higher levels of NT-proBNP vs control $(160,7[88,6-335,4]$ vs $55,2[36,6-70,3] \mathrm{pg} / \mathrm{ml}, p<0,001)$. Elevated levels of NTproBNP (>125,0 pg/ml) was detected in $18(64 \%)$ SLE pts. In SLE pts NT-proBNP serum levels showed positive correlation with creatinine $(\mathrm{r}=0,480, p<0,01)$, uric acid $(r=0,427, p<0,05)$, ACL lgG $(r=0,710, p<0,001)$, antiDNA $(r=0,395, p<0,05)$, ANA levels $(r=0,256, p<0,05)$, left ventricle (LV) end-systolic dimension ( $r=0,442$, $p<0,05)$, mean pulmonary artery pressure $(r=0,486, p<0,05)$; and negative correlation with hemoglobin level $(\mathrm{r}=-0,493, p<0,01), \mathrm{C} 4$ complement component $(\mathrm{r}=-0,475, p<0,05)$, glomerular filtration rate $(\mathrm{r}=-0,558, p<0,01)$ and LV ejection fraction $(r=-0,505, p<0,01)$; left ventricular diastolic dysfunction (DDLV) was only in pts with NT-proBNP levels $>125,0 \mathrm{pg} / \mathrm{ml}$. Mean NT-proBNP concentration in verified DDLV cases $(n=5(18 \%))$ considerably exceeded normal values, reaching up to $799,2[276,6-1777,0] \mathrm{pg} / \mathrm{ml}$.

Conclusions: Untreated SLE patients without a history of myocardial infarction, coronary procedure or any evidence of heart failure demonstrated higher NTproBNP concentration as compared to healthy controls $(p<0,001)$. NT-proBNP levels showed correlation with numerous SLE markers (ACL IgG, ANA, antiDNA, C4 fragment of complement), kidney function (creatinine, uric acid, glomerular filtration rate) and myocardial function (end-systolic dimension of the LV, mean pulmonary artery pressure, LV ejection fraction). No correlation was documented between NT-proBNP concentration and TRF or inflammatory markers (CRP, IL-6, INF- $\alpha$ ). All abovementioned data suggest presumable SLE-associated autoimmune damage of cardiomyocytes and/or mediated decrease of myocardial function caused by kidney disease.

Disclosure of Interest: None declared

DOI: 10.1136/annrheumdis-2017-eular.3735

\section{FRI0300 NEUROLOGICAL INVOLVEMENT IN PRIMARY SJÖGREN SYNDROME}

T. Ben Salem ${ }^{1,1,1}$, I. Naceur, M. Tougorti, M. Lamloum, I. Ben Ghorbel, M.H. Houman. Internal Medicine, Rabta university hospital, Tunis, Tunisia

Background: Prevalence of neurological involvements (NI) in primary Sjögren Syndrome (pSS) varies from 10 to $60 \%$ and depends on whether they are screened systematically or only when they are symptomatic.

Objectives: The aim of the study was to describe the prevalence, clinical patterns and treatment of $\mathrm{NI}$ in pSS.

Methods: We performed a retrospective study of patients with pSS (AmericanEuropean Consensus Group criteria) and followed in an internal medical department over a period of 15 years. Patients with NI were enrolled after excluding other potential causes. We did investigate neurologic systems only when patients present with symptoms.

Results: Primary Sjögren Syndrome was diagnosed in 155 patients, 41 had neurological manifestations (26.4\%). They were 5 male and 36 female. The mean age at disease onset was $49+/-13$ years. The average delay from $\mathrm{NI}$ onset to pSS diagnosis was four months for peripheral nervous system (PNS) and 12 
months for central nervous system (CNS). NI revealed the disease in 14 cases (34\%). CNS and PNS involvements were respectively found in 27 and 19 cases (six patients had both PNS and CNS). In patients with CNS disorders, headache was the most frequent symptom $(n=11)$. Pyramidal syndrome was found in 9 cases and cerebellar syndrome in one patient. Aseptic meningitis was noted in two cases. Brain MRI was performed in 22 cases and showed abnormalities in $82 \%$ of cases. T2 and flair weighted hyperintensities were periventricular $(n=11)$, subcortical $(n=5)$, frontal $(n=3)$ and parietal $(n=2)$. Myelitis was found in one patient.

Patients with PNS showed paresthesia (92\%), motor deficit (22\%) and decreased tendon reflex (18\%). Sensory-motor neuropathy was described in 4 cases, pure sensory neuropathy in 6 cases (including one case of small fiber neuropathy) and motor neuropathy in one patient. Mononeuropathy multiplex was found in 4 cases and polyradiculoneuritis in one patient. Neuromuscular biopsy was performed in five patients and showed vasculitis in two cases. Trigeminal nerve and cochlear nerve were respectively involved in four and one patients. Antinuclear antibodies, anti-SSA and anti-SSB antibodies were respectively positive in $71 \%, 50 \%$ and $28 \%$ of cases. $\mathrm{NI}$ were treated with corticosteroids and immunosuppressive therapy in respectively $56 \%$ and $41 \%$ of cases. Outcome was good in $48 \%$ of the patients. Comparison of clinical and biological features in patients with and without $\mathrm{NI}$ showed no significant differences, only fatigue was significantly associated to $\mathrm{NI}(45.5 \%$ vs $12.9 \%$; $\mathrm{p}=0.005)$.

Conclusions: In our series, NI prevalence was similar to other groups.

CNS involvements were more frequent than PNS manifestations whereas in other ethnic groups they are less frequent.

Disclosure of Interest: None declared

DOI: 10.1136/annrheumdis-2017-eular.2772

\section{FRI0301 CHARACTERIZATION OF FEVER IN HOSPITALIZED PATIENTS WITH SYSTEMIC LUPUS ERYTHEMATOSUS: LESSONS FROM THE JIANGSU COHORT}

X. Feng ${ }^{1}$, W. Pan ${ }^{2}$, L. Liu ${ }^{3}$, M. Wu ${ }^{4}$, L. Sun ${ }^{1}$ on behalf of Jiangsu Lupus Collaborative Group. ${ }^{1}$ Department of Rheumatology, The Affiliated Drum Tower hospital of Nanjing University Medical School, Nanjing; ${ }^{2}$ Department of Rheumatology, Huaian First People's Hospital, Huaian; ${ }^{3}$ Department of Rheumatology, Xuzhou No. 4 People's Hospital, Xuzhou; ${ }^{4}$ Department of Rheumatology, The 3rd Affiliated Hospital of Suzhou University, Changzhou, China

Background: Fever is one of the main symptoms leading to the admission of patients with systemic lupus erythematosus (SLE) and its causes are usually complicated.

Objectives: To explore the prevalence and clinical characteristics of fever in Chinese SLE patients at the time of their first admission and to find out whether fever is related to a poor prognosis.

Methods: A follow-up study aimed to delineate SLE prognosis had been conducted by our Lupus Collaborative Group under the supervisor of Jiangsu Rheumatology Association to collect the data of patients who had ever recorded first admissions during the 1999-2009 decade (1). Among which, those with admission temperature documented were extracted for the assessment of potential factors associated with fever. The independency of various clinical features linked to fever was established by binary logistic regression analysis, and the relation between fever and patient's survival was determined by Cox regression analysis. Results: Totally 1,348 patients were enrolled, in which 1,049 (77.8\%) had normal temperature, $221(16.4 \%)$ had low and moderate fever and $78(5.8 \%)$ had high fever at the time of their first hospitalization. Compared with those having normal temperature, fever patients were more likely to have short disease duration (RR 0.74), concurrent infection (RR 3.29) and gastrointestinal (RR 1.57) as well as hematological involvements (RR 1.56). High C reactive protein level (RR 2.08) and positive anti-Sm antibody (RR1.55) were the two laboratory factors related to fever at admission. However, age, gender, SLEDAI score or erythrocyte sedimentation rate was not independently associated with fever in this cohort. Cox regression analysis showed that there was no correlation between fever and overall deaths (Figure 1) as well as infection induced deaths.

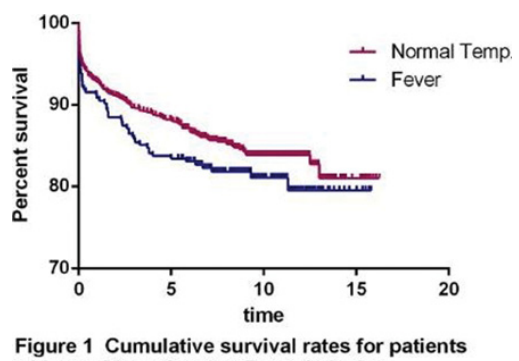

with or without fever at first admission

Conclusions: Both infection and lupus itself (especially gastrointestinal and hematological involvements) contribute to fever in SLE patients. Although the presence of fever increases the complexity of disease treatment, it does not constitute a risk factor for patient's long-term outcome.

\section{References:}

[1] Feng X, Pan W, Liu L, et al. Prognosis for hospitalized patients with systemic lupus erythematosus in China: 5-year update of the Jiangsu cohort. PLoS One. 2016;11(12):e0168619.

Acknowledgements: This study was supported by National Natural Science Foundation of China (81373198) and Jiangsu Provincial Special Program of Medical Science (BE2015602).

Disclosure of Interest: None declared

DOI: 10.1136/annrheumdis-2017-eular.3140

\section{FRI0302 ELEVATED CYSTEINE-RICH PROTEIN 61 IN SYSTEMIC LUPUS ERYTHEMATOSUS-ASSOCIATED PULMONARY ARTERIAL HYPERTENSION}

$\underline{\text { Y. Fan }}{ }^{1}$, J. Qian ${ }^{2}$, Y. Hao ${ }^{1}$, M. Li ${ }^{2}$, L. Gao ${ }^{3}$, X. Zeng ${ }^{2}$, Z. Zhang ${ }^{1} .{ }^{1}$ Departmen of Rheumatology and Clinical Immunology, Peking University First Hospital;

${ }^{2}$ Department of Rheumatology, Peking Union Medical College Hospital, Peking Union Medical College \& Chinese Academy of Medical Sciences, Key Laboratory of Rheumatology and Clinical Immunology, Ministry of Education; ${ }^{3}$ Department of Rheumatology, Beijing Shijitan Hospital, Capital Medical University, Beijing, China

Background: Pulmonary arterial hypertension (PAH) is a complex and devastating complication of connective tissue diseases that leads to severe morbidity and mortality.Unlike in Caucasians, it is systemic lupus erythematosus that recognized as the the major underlying cause of CTD associated PAH in Asian countries, especially in China [1]. Early diagnosis and intervention is vital for better long-term outcome in SLE-PAH patients.Previous study has demonstrated Cysteine-rich protein 61 (Cyr61) was highly expressed in SLE patients [2]. However,the role of Cyr61 in pulmonary arterial hypertension $(\mathrm{PAH})$ remains unknown.

Objectives: To explore the value of Cyr61 for PAH in SLE patients by comparing the plasma Cyr61 levels in SLE patients with/without PAH.

Methods: Plasma samples from two tertiary medical centers were obtained from 54 patients with definite SLE-PAH, 52 age,gender and SLEDAI matched SLE patients without $\mathrm{PAH}$, and 54 age and gender matched healthy controls. Plasma Cyr61 concentration was measured by enzyme-linked immunosorbent assay. Results: Plasma Cyr61 concentration in SLE-PAH patients was significantly higher than the matched SLE patients and healthy controls (median (IQR): 172.5 (143.8, 218.2), 124.9 (104.1, 154.7), 58.17 (28.9, 80.4) respectively, $\mathrm{P}<0.001$ ) (Figure1). The sensitivity and specificity of Cyr61 in predicting the presence of PAH in entire SLE patients were $79.6 \%$ and $67.3 \%$. Receiver operating characteristic curve analysis showed the area under the curve was 0.757 (95\% Cl: 0.662-0.852), with $140.6 \mathrm{pg} / \mathrm{ml}$ as the cut off concentration (Figure2). Further multivariate logistic regression analyses revealed high Cyr61 level $(>140.6)$ is an independent risk factor for SLE patients to develop PAH (OR:7.822 (95\% Cl: 2.224-41.138)) (Table 1). Additionally, weak to moderate positive correlations were observed between Cyr61 concentration and serositis, hematological involvement, red blood cell distribution width, right ventricular systolic pressure and right ventricular diameter measured by echocardiography in entire SLE population.
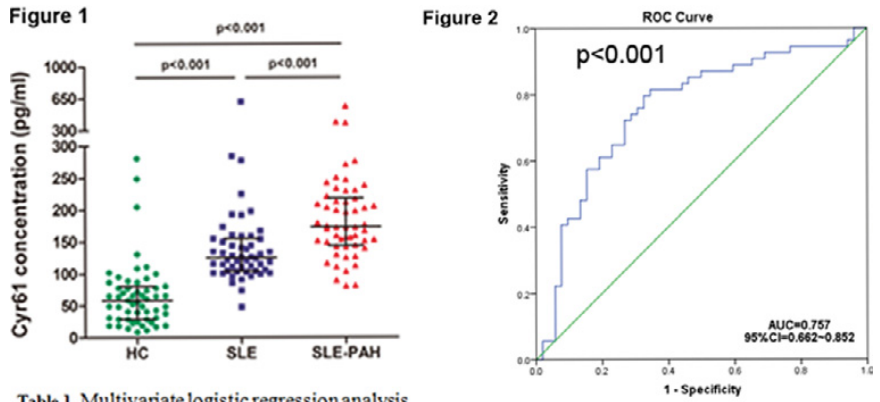

Table 1. Multivariate logistic regression analysis

\begin{tabular}{lcccc}
\hline \multicolumn{1}{c}{ Variables } & OR & \multicolumn{2}{c}{$95 \% \mathrm{CI}$} & P value \\
\hline High Cyr61 level & 7.822 & 2.224 & 41.138 & 0.002 \\
Serositis & 8.244 & 1.854 & 36.663 & 0.006 \\
Anti-RNPpositivity & 3.902 & 1.067 & 14.267 & 0.040 \\
Renal disorders & 0.174 & 0.044 & 0.689 & 0.013 \\
Anti-dsDNA positivity & 0.171 & 0.046 & 0.636 & 0.008 \\
\hline
\end{tabular}

Conclusions: Plasma Cyr61 level was significantly higher in SLE-PAH patients than SLE patients without PAH. Cyr61 may be used as a biomarker for PAH complication in SLE patients.

\section{References:}

[1] Hao, Y.J., et al., Connective tissue disease-associated pulmonary arterial hypertension in Chinese patients. European Respiratory Journal, 2014. 44(4): p. 963-972.

[2] Lin, J., et al., Serum Cyr61 is associated with clinical disease activity and inflammation in patients with systemic lupus erythematosus. Medicine (Baltimore), 2015. 94(19): p. e834 